\title{
Elaboração de farofa de grãos, sementes oleaginosas e castanha de caju: composição de fibras, ácidos graxos e aceitação
}

\author{
Development of a "farofa" $\left(^{*}\right)$ containing grains, oilseeds and cashew nut: \\ fatty acid \& fibre composition and acceptance
}

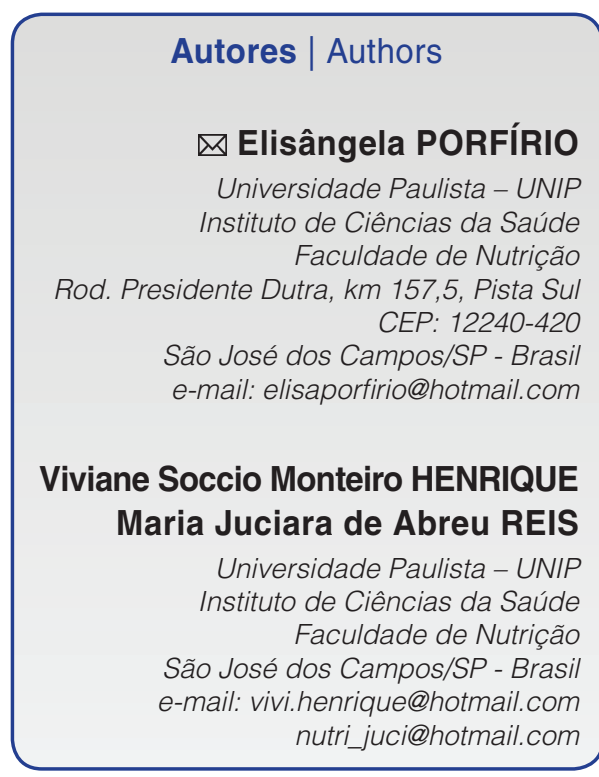

$\triangle$ Autor Correspondente / Corresponding Author

Recebido: Ago. 16, 2013

Aprovado: Ago. 27, 2014

(*) toasted manioc or maize flour mixture

\section{Resumo}

O trabalho objetivou a elaboração de farofa salgada, composta de sementes oleaginosas e castanha de caju, bem como a avaliação de seus atributos nutricionais, comparando-os às recomendações atuais de ingestão e sua aceitação sensorial. As matérias-primas utilizadas na elaboração da farofa foram: gergelim, linhaça dourada, girassol, soja tostada, aveia, flocos de milho, gérmen de trigo, castanha de caju, farinha de milho, óleo de girassol, tempero desidratado e sal. A análise sensorial foi realizada por 120 avaliadores, voluntários, não treinados, de ambos os sexos, frequentadores do campus. A análise físico-química foi realizada considerando teores de fibra alimentar total, solúvel, insolúvel; ácidos graxos; gorduras saturadas, monoinsaturadas, poli-insaturadas, trans; índice de peróxidos e estabilidade oxidativa. Quanto à fibra total, a porção de $30 \mathrm{~g}$ apresentou 11\% dos valores diários recomendados; ácidos graxos $\omega-6$ e $\omega$-3, com proporção de 5:1, razão adequada segundo as recomendações da Organização Mundial de Saúde; ácido graxo oleico representou $15 \mathrm{~g}$ da composição lipídica do produto. Quanto à estabilidade, a farofa mostrou-se estável pelo método Rancimat e índice de peróxido aceitável pela legislação brasileira. Apresentou excelente aceitação entre os avaliadores, com 98\% de aprovação e 89\% de intenção de compra.

Palavras-chave: Fibra dietética; MUFA; PUFA.

\section{Summary}

The objectives of the research were to elaborate a savoury "farofa" ${ }^{*}$ ) containing oil seeds and cashew nuts, and also evaluate its nutritional attributes, comparing them with those of the current dietary intake recommendations. The sensory acceptance was also determined. The raw materials used in the preparation of the farofa were: sesame; golden flaxseed; sunflower; toasted soybeans; oats; corn flakes; wheat germ; cashew nut; corn flour; sunflower oil; dehydrated seasoning and salt. The sensory analysis was carried out by 120 untrained volunteers of both sexes, who regularly frequented the campus. A physicochemical analysis was also carried out determining the total dietary fibre content as well as the soluble and insoluble fibre contents; fatty acids; saturated fats, monounsaturated fats, polyunsaturated fats, trans fats; peroxide index and oxidative stability. As for total fibre, a $30 \mathrm{~g}$ portion presented $11 \%$ of the recommended daily values; the $\omega-6$ and $\omega-3$ fatty acids showed a ratio of 5: 1, which is adequate according to the recommendations of the World Health Organization; and oleic acid represented $15 \mathrm{~g}$ of the lipid composition of the product. The mixture was stable according to the Rancimat method and the peroxide index was acceptable according to Brazilian legislation. The product showed excellent acceptance amongst the volunteers, with 98\% of approval and $89 \%$ of purchasing intention.

Key words: Dietary fiber; MUFA; PUFA. 


\section{Introdução}

Desde o início da história, principalmente com Hipócrates, observou-se que os alimentos possuem características e qualidades capazes de interferir na saúde dos indivíduos (LIRA et al., 2009). A partir do século XX, com métodos analíticos modernos e com a identificação de componentes ativos, além de nutrientes, foi possível determinar como esses constituintes podem contribuir também para a prevenção da manifestação de algumas doenças (BIDLACK e WANG, 2009), sendo a alimentação fator determinante na promoção da saúde (RAIZEL et al., 2011).

Atualmente, o aumento da consciência dos consumidores e a incessante busca por melhor qualidade de vida induz a indústria alimentícia a buscar alimentos para fins especiais (MARINS et al., 2011). Tais expectativas estão voltadas para alimentos com compostos que promovam a saúde e o bem-estar nutricional da população, além da redução do risco de doenças (MORAES e COLLA, 2006).

Inúmeros trabalhos científicos têm sido realizados a respeito do papel das fibras dietéticas no organismo humano. As fibras alimentares são consideradas alimentos funcionais, pois aceleram o trânsito intestinal, prevenindo a obstipação e riscos de câncer de cólon; doenças cardiovasculares; hiperlipidemia; e diabetes (BRASIL, 2005a).

Os ácidos graxos insaturados possuem propriedades anti-inflamatórias, antitrombóticas, antiarrítmicas, hipolipidêmicas e vasodilatadoras, além disso, podem reduzir o colesterol no sangue e contribuir na prevenção da aterosclerose (PATHAK et al., 2014).

O corpo humano produz endogenamente tanto os ácidos graxos saturados como os insaturados, a partir de carboidratos e proteínas, exceto pelo ácido linoleico (C 18:2, ômega 6) e pelo ácido alfa-linolênico (C 18:3, ômega 3), considerados essenciais, devendo eles ser provir da alimentação. Os ácidos graxos ômega 3 e 6 têm como alimentos fontes peixes de água fria, óleos vegetais, sementes oleaginosas, nozes e castanhas (PHILIPPI, 2008).

A farofa é uma preparação culinária tipicamente brasileira, servida como acompanhamento de diversos tipos de pratos, tendo como base principal a farinha de mandioca ou de milho. Além de ter baixo custo na elaboração, é difundida em todo país, fazendo parte da alimentação diária de muitos brasileiros.

Considerando as propriedades nutricionais dos ácidos graxos monoinsaturados e poli-insaturados e das fibras solúveis e insolúveis, bem como o potencial benefício desses à saúde, este trabalho teve como objetivo elaborar uma farofa salgada, composta de sementes oleaginosas e castanha de caju; analisar seus atributos nutricionais e compará-los às recomendações de ingestão diária; e avaliar a sua aceitabilidade sensorial.

\section{Material e método}

\subsection{Elaboração da farofa}

A farofa foi obtida a partir dos ingredientes: óleo de girassol, semente de gergelim, semente de linhaça dourada, semente de girassol, grãos de soja tostados, flocos de aveia, flocos de milho, gérmen de trigo tostado, castanha de caju, farinha de milho, tempero desidratado (salsa, cebola, alho) e sal. As matérias-primas utilizadas no desenvolvimento da farofa foram adquiridas no Mercado Municipal de São Paulo, SP, e no comércio local do município de Jacareí, SP.

O trabalho foi conduzido no Laboratório de Técnica Dietética da Universidade Paulista, campus São José dos Campos, SP. Os ingredientes utilizados no preparo (Tabela 1) foram pesados em uma balança semianalítica calibrada, série BK, marca Gehaka. A castanha de caju foi fragmentada em pedaços médios com faca de aço inoxidável e as sementes de linhaça, girassol e soja foram trituradas na função "pulsar" de um liquidificador doméstico. Todos os ingredientes foram homogeneizados com o auxílio de uma colher de náilon e submetidos à cocção por calor seco pelo período de 5 minutos.

\subsection{Análise de composição química}

As determinações físico-químicas da farofa, como fibra alimentar total, fibra solúvel, fibra insolúvel, composição em ácidos graxos, índice de peróxido e estabilidade oxidativa, foram realizadas pelo Centro de Ciência e Qualidade de Alimentos (CCQA) do Instituto de Tecnologia de Alimentos - ITAL, conforme metodologias baseadas na literatura, sendo: fibra alimentar total - determinação pelo método enzimático/

Tabela 1. Formulação em proporção percentual dos ingredientes da farofa.

\begin{tabular}{lc}
\multicolumn{1}{c}{ Ingredientes } & $\begin{array}{c}\text { Proporção } \\
\text { (\%) }\end{array}$ \\
\hline Óleo de girassol & 3,0 \\
Semente de gergelim & 8,0 \\
Semente de linhaça dourada & 15,0 \\
Semente de girassol & 15,0 \\
Grãos de soja tostados & 7,0 \\
Flocos de aveia & 7,0 \\
Flocos de milho & 4,0 \\
Gérmen de trigo tostado & 4,0 \\
Castanha de caju & 20,0 \\
Farinha de milho amarela & 15,0 \\
Tempero desidratado (salsa, cebola, alho) & 1,5 \\
Sal & 0,5 \\
\hline
\end{tabular}


gravimétrico de Prosky et al. (1984), Horwitz (2006a); fibra solúvel - determinação pelo método de Horwitz (2006b), Prosky et al. (1992); fibra insolúvel - determinação pelo método de Horwitz (2006b), Prosky et al. (1992); composição em ácidos graxos - determinação por cromatografia gasosa (FIRESTONE, 2009a; HORWITZ, 2010a; HARTMAN e LAGO, 1973; MCCANCE e WIDDOWSON, 2002), incluindo gorduras saturadas, monoinsaturadas, poli-insaturadas, trans, ômega 3 e ômega 6; lipídeos totais - determinação pelo método de Horwitz (2010b); índice de peróxidos - determinação pelo método de Firestone (2009b), expresso em miliequivalentes de oxigênio ativo por kg da fração lipídica; estabilidade oxidativa - determinação pelo método de Firestone (2009c), expressa em horas.

\subsection{Análise sensorial}

O estudo contou com a participação de 120 avaliadores não treinados, voluntários, de ambos os sexos, elencados dentre servidores, alunos e visitantes do campus. A análise sensorial ocorreu nas dependências da universidade, no Laboratório de Análise Sensorial anexo ao Laboratório de Técnica Dietética, cujas características obedecem ao seguinte padrão: cabines individuais, de cor neutra e sem comunicação entre si; luminosidade próxima à natural; temperatura entre $18^{\circ} \mathrm{C}$ e $22^{\circ} \mathrm{C}$; umidade relativa entre $60 \%$ a $70 \%$; sem ruídos e odores estranhos (NORONHA, 2003).

Conforme Resolução n. 196/96, de 10 de outubro de 1996 do Conselho Nacional de Saúde (BRASIL, 1996), o protocolo experimental desta pesquisa foi submetido à análise pelo Comitê de Ética em Pesquisa (CEP) da instituição e aprovado sob CAAE n.03677112.0.0000.5512. Todos os participantes assinaram o formulário TCLE (Termo de Consentimento Livre e Esclarecido).

Para determinar a aceitabilidade da formulação foi aplicado o Teste Afetivo que expressa o grau de gostar ou desgostar do produto quanto à impressão de características globais, como aroma, textura e sabor (DUTCOSKY, 2007). As fichas, segundo modelos da NBR 14141 (ABNT, 1998), foram compostas por escala hedônica estruturada de nove pontos, abrangendo de "desgostei muitíssimo" (nota 1) a "gostei muitíssimo" (nota 9), e a escala de intenção de compra, por cinco pontos, de "certamente não compraria" (nota 1) a "certamente compraria" (nota 5). O limite inferior da média para a aceitação do produto foi estabelecido em 6 - "gostei ligeiramente" e 4 - "provavelmente compraria".

Os avaliadores foram servidos com uma bandeja contendo uma amostra do alimento em quantidade padronizada em uma colher de sopa (30 g); uma colher de sobremesa descartável e um copo descartável contendo
$100 \mathrm{~mL}$ de água. Cada participante relacionou a amostra com um valor de acordo com sua preferência.

\section{Resultados e discussão}

\subsection{Fibras}

Para a fibra alimentar total (Tabela 2), uma porção de $30 \mathrm{~g}$ da farofa poderá suprir $11 \%$ das necessidades de ingestão diária de fibras que, de acordo com recomendação do Guia Alimentar para a População Brasileira, elaborado pelo Ministério da Saúde, é de no mínimo 25 g (BRASIL, 2005a). Segundo o Departamento de Agricultura dos EUA, a recomendação de fibra alimentar para indivíduos com risco para diabetes tipo 2 é de $14 \mathrm{~g} / 1000 \mathrm{kcal}$ por dia (AMERICAN DIABETES ASSOCIATION, 2012), para a Sociedade Brasileira de Cardiologia, a recomendação de ingestão de fibra alimentar total para adultos é de $20 \mathrm{~g}$ a $30 \mathrm{~g} / \mathrm{dia}$ (SPOSITO et al., 2007).

As frações das fibras são agrupadas conforme seus componentes e, de acordo com a solubilidade em água, as fibras alimentares são classificadas em fibras solúveis e fibras insolúveis, as quais exercem efeitos fisiológicos distintos no organismo com base na solubilidade (MIRA et al., 2009; BERNAUD e RODRIGUES, 2013).

Dietas ricas em fibras apresentam maior volume e menos densidade calórica e sua presença no estômago promove sensação de saciedade. As fibras insolúveis associadas à ingestão de água regularizam o trânsito intestinal porque aumentam o volume das fezes e normalizam os movimentos intestinais. As fibras solúveis tornam-se viscosas e interferem na absorção de ácidos biliares pelo íleo e, consequentemente, na redução do LDL colesterol que é retirado do sangue para suprir os ácidos biliares perdidos nas fezes (MARLETT et al., 2002).

Os efeitos dos componentes da dieta sobre a incidência de diabetes tipo 2 ainda são desconhecidos, porém sabe-se que a fibra dietética pode fornecer proteção contra a doença por dois mecanismos: pela relação entre a fibra solúvel e a digestão e absorção lenta de carboidratos que retardam a excreção de insulina, evitando picos glicêmicos; pela relação entre fibra insolúvel e o rápido trânsito intestinal, que permite menos tempo de contato entre carboidratos e jejuno (MONTONEN et al., 2003).

Tabela 2. Valores de fibra alimentar total, fibra solúvel e fibra insolúvel (porção de $30 \mathrm{~g}$ ).

\begin{tabular}{lc}
\multicolumn{1}{c}{ Determinação } & (g) \\
\hline Fibra alimentar total (FAT) & $2,7 \pm 0,1$ \\
Fibra alimentar solúvel & $0,7 \pm 0,1$ \\
Fibra alimentar insolúvel & $2,0 \pm 0,2$ \\
\hline
\end{tabular}


A ação protetora da fibra dietética contra o câncer de cólon relaciona-se com o aumento do volume fecal e menor tempo do trânsito intestinal, que permitem reduzir o contato e a interação de agentes mutagênicos fecais com o epitélio intestinal (SLAVIN et al., 1999). Além disso, a fibra solúvel possui alto grau de fermentação no intestino, produzindo ácidos graxos de cadeia curta que, de acordo com estudos, exercem funções importantes na fisiologia do intestino, como melhora do fluxo sanguíneo, aumento da absorção de água e de sódio e diminuição do pH, prevenindo infecções (LONDERO et al., 2009).

\section{2 Ácidos graxos}

Com relação à composição centesimal, a amostra analisada apresentou $37,19 \mathrm{~g}$ de lipídios totais, sendo 5,07 $\mathrm{g}$ de ácidos graxos saturados, 15,39 g de monoinsaturados, $15,07 \mathrm{~g}$ de poli-insaturados e isômeros trans inferiores a 0,01 g. A composição em ácidos graxos está expressa na Figura 1.

As recomendações dietéticas atuais dos profissionais de saúde estimulam a redução do consumo de ácidos graxos saturados (SFA), porém tais orientações não destacam um nutriente substituto na dieta. Estudos realizados em curto prazo demonstraram que a substituição dos ácidos graxos saturados pelos ácidos graxos poli-insaturados pode reduzir os níveis de LDL-C, sendo esse um grande preditor de risco de doença coronariana, sem reduzir de maneira significativa os níveis de HDL-c. O consumo de ácidos graxos poli-insaturados (PUFA) pode também diminuir a resistência à insulina e reduzir a inflamação sistêmica. Esses efeitos sobre fatores de risco sugerem que PUFA pode ser um substituto para SFA na população (MOZAFFARIAN et al., 2010).

Com base em estudos como o de MOZAFFARIAN et al. (2010), a OMS/FAO (2008) atualizou suas referências para o consumo de ácidos graxos,

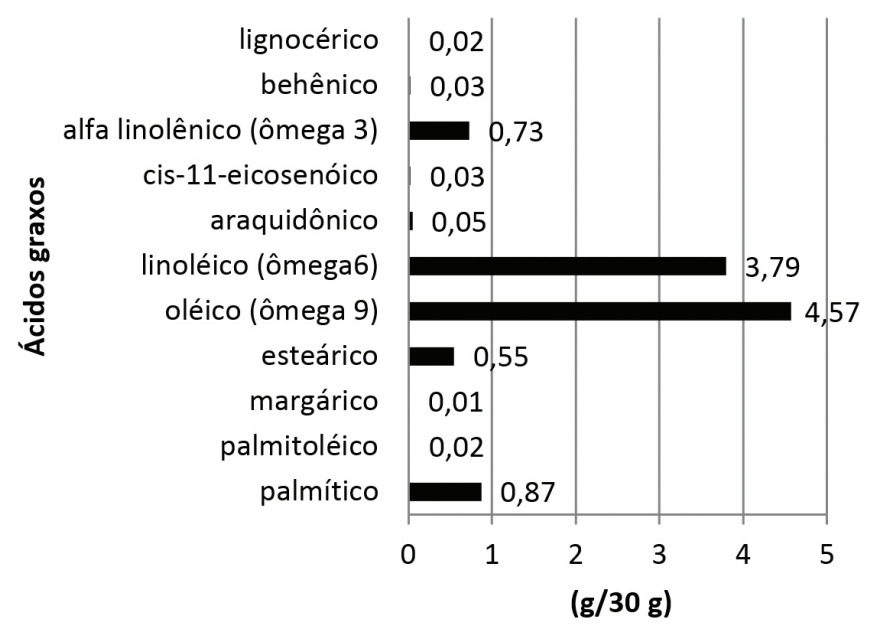

Figura 1. Perfil de ácidos graxos na fração lipídica da farofa com sementes oleaginosas e castanha de caju. aumentando a recomendação de PUFA para $6 \%$ a 11\% do valor calórico total da dieta, com alegações de que existem evidências de que a substituição de gorduras saturadas por poli-insaturadas reduz o colesterol sérico e, consequentemente, o risco de doenças cardiovasculares.

A OMS (2008) orienta ingestão máxima de 35\% de gorduras totais, sendo ácidos graxos saturados $<10 \%$ das calorias totais; ácidos graxos poli-insaturados, de $6 \%$ a 11\%; ácidos graxos monoinsaturados por diferença, sem ultrapassar $20 \%$ das calorias totais; e ácidos graxos trans $<1 \%$. De acordo com a IV Diretriz Brasileira Sobre Dislipidemias e Prevenção da Aterosclerose do Departamento de Aterosclerose da Sociedade Brasileira de Cardiologia (SPOSITO et al., 2007), as recomendações nutricionais para o tratamento das hipercolesterolemias são estimadas como consumo de gordura total entre $25 \%$ a 35\%; ácidos graxos saturados, $<7 \%$; ácidos graxos poliinsaturados, $\leq 10 \%$; e ácidos graxos monoinsaturados, $\leq 20 \%$ das calorias totais.

A OMS/FAO (2008) estabelece a ingestão de $\omega$-6 e $\omega$-3 na razão de 5:1 a 10:1. O Institute of Medicine (IOM, 2005) através da Dietary Reference Intakes (DRI), estabelece nível de ingestão para os ácidos graxos conforme idade, gênero e estado fisiológico, como gestação e lactação. Para homens de 19 a 50 anos, a ingestão de $\omega-6$ deve ser de $17 \mathrm{~g} / \mathrm{dia}$, enquanto que para mulheres da mesma idade a ingestão deve corresponder a $12 \mathrm{~g} /$ dia. Quanto à ingestão de $\omega$-3 são recomendados 1,6 g/dia para homens e 1,1 g/dia para mulheres.

Assim, com relação ao produto desenvolvido neste trabalho, no que se refere aos ácidos graxos $\omega-6$ e $\omega-3$, o resultado obtido quanto à proporção foi de $5: 1$, atingindo a faixa de valores recomendados pela OMS. De acordo com as recomendações da DRI, uma porção de $30 \mathrm{~g}$ da farofa representaria $22 \%$ e $45 \%$ do necessário, para homens; e 31\% e 66\% do necessário para mulheres, de ácidos graxos $\omega-6$ e $\omega-3$, respectivamente.

Além de serem uma importante fonte de energia, os ácidos graxos têm ação vasodilatadora e possível ação na prevenção e/ou tratamento do câncer (mama, próstata e cólon), da depressão e do mal de Alzheimer, na redução da incidência de aterosclerose, apresentando atividade anti-inflamatória, anticoagulante e antiagregante (MESQUITA et al., 2011) e participando da transferência de oxigênio para a corrente sanguínea, da síntese de hemoglobina, da transmissão de impulsos nervosos e da função cerebral, e suas deficiências estão relacionadas à fadiga, problemas dermatológicos, circulatórios e imunológicos, distúrbios gastrointestinais, artrite reumatoide, agravamento de câncer de mama, câncer de próstata; entre outros (YEHUDA et al., 2002).

Quanto ao ácido graxo oleico, representa $15 \mathrm{~g}$ (41\%) da composição lipídica do produto. O ácido graxo oleico é um ácido graxo monoinsaturado e dele deriva o 
Elaboração de farofa de grãos, sementes oleaginosas e castanha de caju: composição de fibras, ácidos graxos e aceitação PORFÍRIO, E. et al.

ômega-9. Ao contrário do ômega-3 e ômega-6, o ácido graxo ômega-9 é produzido pelo corpo e, portanto, não é essencial. As principais fontes de ômega-9 são o azeite de oliva, azeitonas, abacate, amêndoas, amendoim, óleo de gergelim, nozes, pistache, castanha de caju, avelã, macadâmia, entre outras, e ele ocorre naturalmente em maiores quantidades do que qualquer outro ácido graxo (WIN, 2005). O ácido oleico, comparado à gordura saturada, reduz a concentração plasmática de LDL-colesterol sem reduzir os níveis de HDL-colesterol, além disso, induz menor síntese endógena de colesterol. A ingestão regular de ômega-9 está associada à redução do risco de doenças cardiovasculares, aterosclerose e à prevenção de câncer (LOTTENBERG, 2009), diabetes e obesidade (RODRIGUES, 2001).

\subsection{Estabilidade oxidativa e índice de peróxido}

Óleos e gorduras animais ou vegetais e produtos derivados podem sofrer alterações, as quais podem ser percebidas sensorialmente, mesmo em estágios iniciais. A oxidação lipídica é um processo intensivamente estudado, pois esse tipo de deterioração é responsável pela formação de sabores estranhos que reduzem a qualidade e a vida útil dos alimentos, bem como produzem compostos tóxicos com implicações nutricionais indesejáveis (MILANI, 2010; MANCIO, 2011).

Pela legislação brasileira, ANVISA (BRASIL, 2005b), o índice de peróxido para óleos prensados a frio não refinados não pode ser superior a 15 meq/kg, portanto foi verificado que a farofa possui valor aceitável, com 3,45 meq/kg.

O comportamento oxidativo do produto foi medido pelo equipamento Rancimat, sendo a amostra submetida a um período de indução ou tempo necessário para atingir um ponto crítico de oxidação de 18,0 horas. 0 valor apresentando foi satisfatório, pois, de acordo com a norma EN 14112, para que um óleo seja considerado estável deve ser exposto ao teste no Rancimat por um período mínimo de oxidação de 6,0 horas (MOSER, 2009).

Segundo Antoniassi (2001), existem na literatura muitos estudos sobre estabilidade oxidativa de óleos e gorduras, porém para que se estabeleçam comparações são necessárias análises sob mesmas condições de tempo, temperatura, fluxo de ar e quantidade da amostra utilizada. Quanto a esse parâmetro ainda devem ser considerados o alto teor de ácidos graxos insaturados contidos na amostra, bem como a presença de antioxidantes naturais presentes em óleos vegetais como o sesamol, que confere elevada estabilidade química ao óleo de gergelim (BELTRÃO et al., 1994; FIRMINO, 1996).

\subsection{Análise sensorial}

A amostra populacional foi composta por 120 avaliadores, sendo $64 \%$ do gênero feminino e $36 \%$ do masculino. Quanto à faixa etária, 12\% tinham entre 18 e 20 anos; 43\%, entre 21 e 30 anos; 17\%, entre 31 e 40 anos; $13 \%$, entre 41 e 50 anos; $11 \%$, entre 51 e 60 anos; e $5 \%$, entre 61 e 70 anos.

O produto elaborado foi considerado aceito, pois a maioria dos avaliadores, 98\%, atribuíram notas de 6 a 9 e apenas $2 \%$ atribuíram nota inferior a 6 . Com relação à intenção de compra, os resultados demonstraram que $61 \%$ dos provadores certamente comprariam o produto, $28 \%$ provavelmente o comprariam e $11 \%$ não sabem se o comprariam ou não.

Segundo Cardarelli (2006), um produto é considerado aceito quando atinge o índice de aceitabilidade de, no mínimo, 70\%. As características sensoriais são determinantes na escolha do consumidor diante de um novo produto, mesmo que esse apresente conceitos de funcionalidade, com efeitos benéficos à saúde.

\section{Conclusões}

Diante dos resultados apresentados neste trabalho, a farofa de sementes oleaginosas com castanha de caju apresentou altos teores de fibra alimentar e ácidos graxos insaturados, bem como excelente aceitação entre os avaliadores, podendo ser uma alternativa capaz de suprir preferências de consumidores preocupados em incorporar alimentos com características funcionais à dieta. No entanto, a vida útil da farofa, baseada no aparecimento de flavor oxidado deve ser reavaliada a partir da relação entre análise sensorial e diferentes ensaios sob condições predeterminadas de armazenamento.

\section{Referências}

AMERICAN DIABETES ASSOCIATION. Executive Summary: Standards of Medical Care in Diabetes. Diabetes Care, Alexandria, v. 35, n. 1, 2012. Supplement.

ANTONIASSI, R. Métodos de Avaliação da Estabilidade Oxidativa de Óleos e Gorduras. Boletim do Centro de Pesquisa e Processamento de Alimentos, Curitiba, v. 19, n. 2, p. 353-380, 2001. Disponível em: <http://ojs.c3sl.ufpr.br/ ojs/index.php/alimentos/article/view/1243/1043>.

ASSOCIAÇÃO BRASILEIRA DE NORMAS TÉCNICAS - ABNT. NBR 14141: Escalas Utilizadas em Análise Sensorial de Alimentos e Bebidas. Rio de Janeiro: ABNT, 1998.

BELtRÃO, N. E. M.; FREIRE, E. C.; LIMA, E. F. Gergelim Cultura no Trópico Semi-árido Nordestino. Campina Grande: EMBRAPA-CNPA, 1994. 52 p. (Circular Técnica, 18). 
Elaboração de farofa de grãos, sementes oleaginosas e castanha de caju: composição de fibras, ácidos graxos e aceitação PORFÍRIO, E. et al.

BERNAUD, F. S. R.; RODRIGUES, T. C. Fibra Alimentar: Ingestão Adequada e Efeitos sobre a Saúde do Metabolismo. Arquivos Brasileiros de Endocrinologia \& Metabologia, São Paulo, v. 57, n. 6, p. 397-405, 2013. http://dx.doi.org/10.1590/S000427302013000600001

BIDLACK, W. R.; WANG, W. Planejamento de Alimentos Funcionais. In: SHILS, M. E.; SHIKE, M.; ROSS, A. C.; CABALLERO, B.; COUSENS, R. J. Nutrição Moderna na Saúde e na Doença. 10. ed. Barueri: Manole, 2009. p. 1925-1926.

BRASIL. Ministério da Saúde. Conselho Nacional de Saúde. Resolução n 196, de 10 de Outubro de 1996. Aprova as Diretrizes e Normas Regulamentadoras das Pesquisas Envolvendo Seres Humanos. Diário Oficial da União, Poder Executivo, DF, Brasília, 16 out. 1996. Seção 1.

BRASIL. Ministério da Saúde. Guia Alimentar para a População Brasileira: Promovendo a Alimentação Saudável. Brasília: Ministério da Saúde, 2005a.

BRASIL. Agência Nacional de Vigilância Sanitária - ANVISA. Resolução RDC n² 270, de 22 de setembro de 2005. Regulamento Técnico para Óleos Vegetais, Gorduras Vegetais e Creme Vegetal. Diário Oficial da União, Poder Executivo, Brasília, DF, 23 set. 2005b. Disponível em: <http://e-legis.anvisa. gov.br/leisref/public/showAct. php?id=18829\&word=>.

CARDARELLI, R. H. Desenvolvimento de Queijo petit-suisse Simbiótico. 2006. 149 f. Tese (Doutorado em Tecnologia de Alimentos)-Faculdade de Ciências Farmacêuticas, Universidade de São Paulo, São Paulo, 2006. Disponível em: <file:///C:/Users/ Elis/Downloads/tese_doutorado_Haissa_R_Cardarelli_2006_ sem_ata.pdf $>$.

DUTCOSKY, S. D. Análise Sensorial de Alimentos. 2. ed. Curitiba: Champagnat; 2007.

FIRESTONE, D. (Ed.). Official Methods and Recommended Practices of the American Oil Chemists Society. 6th. ed. Urbana: AOCS, 2009a. Met. Ce 1f-96, Ce 1-62.

FIRESTONE, D. (Ed.). Official Methods and Recommended Practices of the American Oil Chemists Society. 6th. ed. Urbana: AOCS, 2009b. Met. Cd 8b-90.

FIRESTONE, D. (Ed.). Official Methods and Recommended Practices of the American Oil Chemists Society. 6th. ed. Urbana: AOCS, 2009c. Met. Cd 12b-92.

FIRMINO, P. T. Gergelim: Sistemas de Produção e seu Processo de Verticalização, Visando Produtividade no Campo e Melhoria da Qualidade da Alimentação Humana. Campina Grande: EMBRAPA-CNPA, 1996. (Prêmio Jovem Cientista).

HARTMAN, L.; LAGO, R. C. A. Rapid Preparation of Fatty Acid Methyl Esters from Lipids. Laboratory Practice, London, v. 22 , n. 8, p. 475-6, 1973.
HORWITZ, W. (Ed.). Official Methods of Analysis of AOAC International. 18th ed. Gaithersburg: AOAC, 2006a. p. 97-98. cap. 45, met. 985.29.

HORWITZ, W. (Ed.). Official Methods of Analysis of AOAC International. 18th ed. Gaithersburg: AOAC, 2006b. p. 7-10. cap. 32, met. 991.43.

HORWITZ, W. (Ed). Official Methods of Analysis of AOAC International. 18th ed. Gaithersburg: AOAC, 2010a. p. 20. cap. 41, met. 996.06

HORWITZ, W. (Ed). Official Methods of Analysis of AOAC International. 18th ed. Gaithersburg: AOAC, 2010b. p. 5. cap. 32 met. 922.06.

INSTITUTE OF MEDICINE - IOM. Dietary Reference Intakes (DRIs) for Energy, Carbohydrate, Fiber, Fat, Fatty Acids, Cholesterol, Protein, and Amino Acids. Washington: National Academies Press, 2005. (Part 1).

LIRA, C. R. G.; ZUCCO, F; NEGRÃO, A. N.; SILVA, M. A. S.; MURAKAMI, F. S. Nutracêuticos: Aspectos sobre Segurança, Controle de Qualidade e Legislação. Revista Brasileira de Farmácia, Rio de Janeiro, v. 90, n. 1, p. 45-49, 2009.

LONDERO, P. M. G.; RIBEIRO, N. D.; JOST, E.; MAZIERO, S. M.; CERUTTI, T.; POERSCH, N. L. Genética dos Teores de Fibras Insolúvel e Solúvel em Grãos de Feijão. Revista Ceres, Viçosa, v. 56, n. 2, p. 150-155, 2009. Disponível em: <http://www.redalyc. org/pdf/3052/305226730006.pdf>

LOTTENBERG, A. M. P. Importância de Gordura Alimentar na Prevenção e no Controle de Distúrbios Metabólicos e da Doença Cardiovascular. Arquivos Brasileiros de Endocrinologia \& Metabologia, São Paulo, v. 53, n. 5, p. 595-607, 2009.

MANCIO, M. M. Estabilidade dos Ácidos Graxos Poliinsaturados Presentes em Fórmulas Infantis Comerciais. Brazilian Journal of Food Technology, Campinas, v. 14, n. 2, p. 145-53, 2011. Disponível em: <http://bjtt.ital.sp.gov.br/artigos/html/busca/PDF/ v14n2457a.pdf>.

MARINS, B. R.; ARAÚJO, I. S.; JACOB, S. C. A Propaganda de Alimentos: Orientação ou apenas Estímulo ao Consumo? Ciência \& Saúde Coletiva, Rio de Janeiro, v. 16, n. 9, p. 38733882, 2011. Disponível em: <http://www.scielo.br/scielo. php?pid=S1413-81232011001000023\&script=sci_arttext $>$.

MARLETT, J. A.; MCBURNEY, M. I.; SLAVIN, J. L. Health implications of dietary fiber. Journal of the American Dietetic Association, Bethesda, v. 102, n. 7, p. 993-1000, 2002. Disponível em: <http://www.journals.elsevierhealth.com/ periodicals/yjada/article/S0002-8223(02)90228-2/fulltext artg. html>.

MCCANCE, R. A.; WIDDOWSON, E. M. McCance and Widdowson's: The Composition of Foods. Cambridge: Royal Society of Chemistry, 2002. p. 537. 
Elaboração de farofa de grãos, sementes oleaginosas e castanha de caju: composição de fibras, ácidos graxos e aceitação PORFÍRIO, E. et al.

MESQUITA, T. R.; SOUZA, A. A.; CONSTANTINO, E.; PELÓGIA, N. C. C.; POSSO, I. P.; PIRES, O. C. Efeito Anti-inflamatório da Suplementação Dietética com Ácidos Graxos Ômega-3, em Ratos. Revista Dor, São Paulo, v. 12, n. 4, p. 337-41, 2011. Disponível em: <http://www.scielo.br/scielo.php?pid=S180600132011000400010\&script=sci_arttext $>$.

MILANI, L. I. G. Oxidação Lipídica, Características Sensoriais e Cor da Carne de Frango Adicionada de Extratos de Caqui (Diospyros kaki, L.) e Submetida a Tratamento Térmico. Brazilian Journal of Food Technology, Campinas, v. 13, n. 4, p. 242-250, 2010. Disponível em: <http://bjft.ital.sp.gov.br/artigos/html/ busca/PDF/v13n4429a.pdf>.

MIRA, G. S.; GRAF, H.; CÂNDIDO, L. M. B. Visão Retrospectiva em Fibras Alimentares com Ênfase em Beta-glucanas no Tratamento do Diabetes. Brazilian Journal of Pharmaceutical Sciences, v. 45, n. 1, p. 11-20, 2009. Disponível em: <http:// www.scielo.br/pdf/bjps/v45n1/03.pdf artg.html>

MONTONEN, J.; KNEKT, P.; JÄRVINEN, T.; AROMAA, A.; REUNANEN, A. Whole-grain and Fiber Intake and the Incidence of Type 2 Diabetes. American Journal of Clinical Nutrition, Bethesda, v. 77, n. 3, p. 622-629, 2003. Disponível em: <http:// www.ajcn.org/content/77/3/622.full artg.html>

MORAES, F. P.; COLLA, L. M. Alimentos Funcionais e Nutracêuticos: Definições, Legislação e Benefícios à Saúde. Revista Eletrônica de Farmácia, Goiás, v. 3, n. 2, p. 109-122, 2006. http://www.revistas.ufg.br/index.php/REF/article/ viewFile/2082/2024

MOSER, B. R. Comparative Oxidative Stability of Fatty Acid Alkyl Esters by Accelerated Methods. Journal of the American Oil Chemists' Society, Champaign, v. 86, n. 7, p. 699-706, 2009. Disponível em: <http://naldc.nal.usda.gov/download/32987/ PDF>.

MOZAFFARIAN, D.; MICHA, R.; WALLACE, S. Effects on Coronary Heart Disease of Increasing Polyunsaturated Fat in Place of Saturated Fat: a Systematic Review and Meta-analysis of Randomized Controlled Trials. PLoS Med, San Francisco, v. 7, n. 3, 2010. Disponivel em: <http://www.plosmedicine.org/article/ info\%3Adoi\%2F10.1371\%2Fjournal.pmed.1000252>.

NORONHA, J. F. Apontamentos de Análise Sensorial: Análise Sensorial. Metodologia. Versão 1.0. Coimbra: Escola Superior Agrária de Coimbra, 2003. Disponível em: <http://www.esac.pt/ noronha/A.S/Apontamentos/sebenta_v_1_0.pdf>.

ORGANIZAÇÃO MUNDIAL DE SAÚDE - OMS. Interim Summary of Conclusions and Dietary Recommendations on Total Fat \& Fatty Acids. From the Joint FAO/WHO Expert Consultation on Fats and Fatty Acids in Human Nutrition. Geneva: WHO, 2008. p. 10-14.
PATHAK, N.; RAI, A. K.; KUMARI, R.; BHAT, K. V. Value Addition in Sesame: a Perspective on Bioactive Components for Enhancing Utility and Profitability. Pharmacognosy Reviews, Bangalore, v. 6, n. 16, p. 147-155, 2014.

PHILIPPI, S. T. (Org.). Pirâmide dos Alimentos: Fundamentos Básicos da Nutrição. Barueri: Manole, 2008.

PROSKY, L.; ASP, N. G.; FURDA, I.; DEVRIES, J. W.; SCHWEIZER, T. F.; HARLAND, B. F. Determination of Total Dietary Fiber in Foods, Food Products and Total Diets: Interlaboratory Study. Journal of the Association Official Analytical Chemists, Arlington, v. 67, n. 6, p. 1044-1052, 1984.

PROSKY, L.; ASP, N. G.; FURDA, I.; DEVRIES, J. W.; SCHWEIZER, T. F.; LEE, S. C. Determination of Insoluble and Soluble Dietary Fibers in Foods and Food Products. Journal of the Association of Official Analytical Chemists International, Arlington, v. 75, n. 2, p. 360-367, 1992.

RAIZEL, R.; SANTINI, E.; KOPPER, A. M.; REIS FILHO, A. D. Efeitos do Consumo de Probióticos, Prebióticos e Simbióticos para o Organismo Humano. Revista Ciência \& Saúde, Brasília, v. 4, n. 2, p. 66-74, 2011.

RODRIGUES, H. G. Modulação do Processo de Cicatrização pelos Ácidos Oleico e Linoléico. 2001. 91 f. Tese (Doutorado em Fisiologia Humana)-Instituto de Ciências Biomédicas, Universidade de São Paulo, São Paulo, 2001.

SLAVIN, J. L.; MARTINI, M. C.; JACOBS JUNIOR, D. R.; MARQUART, L. Plausible Mechanisms for the Protectiveness of Whole Grains. American Journal of Clinical Nutrition, Bethesda, v. 70, n. 3, p. 459S-63S, 1999. Disponível em: <http:// www.ajcn.org/content/70/3/459S.full.pdf+html artg.html>

SPOSITO, A. C.; CARAMELLI, B.; FONSECA, F. A. H.; BERTOLAMI, M. C. IV Diretriz Brasileira sobre Dislipidemias e Prevenção da Aterosclerose. Departamento de Aterosclerose da Sociedade Brasileira de Cardiologia. Arquivos Brasileiros de Cardiologia, São Paulo, v. 88, p. 2-19, 2007. Suplemento 1. Disponível em: <http://www.scielo.br/scielo.php?pid=S0066782X2007000700002\&script=sci_arttext $>$.

WIN, D. T. Oleic Acid: the Anti-breast Cancer Component in Olive Oil. AU Journal of Technology, Bangkok, v. 9, n. 2, p. 75-8, 2005. Disponível em: <http://www.journal.au.edu/ au_techno/2005/oct05/vol9num2_article02.pdf>

YEHUDA, S.; RABINOVITZ, S.; CARASSO, R. L.; MOSTOFSKY, D. I. The Role of Polyunsaturated Fatty Acids in Restoring the Aging Neuronal Membrane. Neurobiology of Aging, Fayetteville, v. 23, n. 5, p. 843-53, 2002. http://dx.doi.org/10.1016/S01974580(02)00074-X 